\title{
A Note on Center Problems With Forbidden Polyhedra
}

\author{
Horst W. Hamacher* Anita Schöbel*
}

November 10, 1997

\begin{abstract}
The problem of finding an optimal location $X^{*}$ minimizing the maximum Euclidean distance to existing facilities is well solved by e.g.the Elzinga-Hearn algorithm.

In practical situations $X^{*}$ will however often not be feasible. We therefore suggest in this note a polynomial algorithm which will find an optimal location $X^{F}$ in a feasible subset $F \subseteq \mathbb{R}^{2}$ of the plane.
\end{abstract}

* partially supported by grants of the Deutsche Forschungsgemeinschaft and EU Human Capital\& Mobility Program 


\section{Restricted Euclidean Center Problems}

Using the classification scheme introduced in [Ham95] and [HN93] $1 / P / v_{i}=1 / l_{2} / \max$ is the problem of finding

- 1 new location $X^{*}$

- in the plane,

- with constant weights $v_{i}=1$,

- with respect to the Euclidean distance, $l_{2}(X, Y)=\sqrt{\left(x_{1}-y_{1}\right)^{2}+\left(x_{2}-y_{2}\right)^{2}}$

- minimizing the maximum distance to existing facilities $E x_{m}=\left(a_{m 1}, a_{m 2}\right), m=1, \ldots, M$.

We therefore solve the problem

$$
\min _{X \in \mathbb{R}^{2}} \max _{m=1, \ldots, M} l_{2}\left(X, E x_{m}\right)
$$

For $M=2$ and $M=3$ an optimal location $X^{*}$ can be found based on the following geometric observations. Here $L_{i j}$ will denote the line connecting $E x_{i}$ and $E x_{j}$.

For $M=2: \quad X^{*}$ is the center point of the line $L_{12}$

For $M=3$ : Consider the triangle $\triangle$ spanned by $E x_{1}, E x_{2}$ and $E x_{3}$. If $\triangle$ is acute $X^{*}$ is the unique center of the median of $T$, which is found as the intersection point of the perpendicular bisectors of the lines $L_{12}, L_{23}$ and $L_{13}$. If $\triangle$ is obtuse $X^{*}$ is the center point of the hypotenuse of $\triangle$.

For general $M 1 / P / v_{i}=1 / l_{2} / \max$ can be solved by comparing the optimal locations of all groups of three locations $E x_{i_{1}}, E x_{i_{2}}, E x_{i_{3}},\left\{i_{1}, i_{2}, i_{3}\right\} \subseteq\{1, \ldots, M\}\left(\right.$ an $0\left(M^{4}\right)$ algorithm) or by applying the more efficient algorithms of Elzinga-Hearn [EH72] and Megiddo, see [Meg83]. Public domain codes of the former algorithm can be found in [HV95] and [Nic95].

In the restricted location problem $1 / P / v_{i}=1, R$ polyhedron $/ l_{2} / \max$ we assume that some polyhedron $R$ is given, such that the new location $X$ is not allowed to be contained in the interior $\operatorname{int}(R)$ of $R$, i.e. we solve

$$
\min _{X \in F:=\mathbb{R}^{2} \backslash \operatorname{int}(R)} \max _{m=1, \ldots, M} l_{2}\left(X, E x_{m}\right)
$$

This type of situation is very common in practical situations: Restricting sets may, for instance, represent non-suitable regions for the new facility (e.g. natural habitats, lakes) or space taken by existing facilities.

In the next section we will discuss some basic results which will lead to an efficient algorithm described in Section 3. Section 4 contains some information on how to deal with generalizations of this problem. 


\section{Basic Results for Restricted Euclidean Center Prob- lems}

Denote with $X^{*}$ the unique optimal and with $X^{R}$ any optimal location of the unrestricted and restricted problem, respectively. Corresponding optimal objective values are

$$
z^{*}=\max _{m=1, \ldots, M} l_{2}\left(X^{*}, E x_{m}\right)
$$

and

$$
z^{R}=\max _{m=1, \ldots, M} l_{2}\left(X^{R}, E x_{m}\right)
$$

If $X^{*} \in \mathbb{R}^{2} \backslash \operatorname{int}(R)$, then $X^{R}=X^{*}$ and the restricted problem is trivially solved. We therefore assume in the following $X^{*} \in \operatorname{int}(R)$. If $\partial R$ is the boundary of $R$ we know from [HN95]:

Theorem 2.1 $X^{R} \in \partial R$.

Optimal locations $X^{R}$ can be characterized using

and

$$
\text { level curves } L_{=}(z):=\left\{Y: \max _{m=1, \ldots, M} l_{2}\left(E x_{m}, Y\right)=z\right\}
$$

$$
\text { level sets } \quad L_{\leq}(z):=\left\{Y: \max _{m=1, \ldots, M} l_{2}\left(E x_{m}, Y\right) \leq z\right\}
$$

Since $z^{R}=\min \left\{z: L_{\leq}(z) \cap\left(\mathbb{R}^{2} \backslash \operatorname{int}(R)\right) \neq \emptyset\right\}$ we obtain the following result (see [HN95]).

Theorem $2.2 z^{R}$ is the optimal objective value of the restricted Euclidean center problem if and only if

$$
\begin{array}{ll}
\text { (1) } L_{\leq}\left(z^{R}\right) \subseteq R & \text { and } \\
\text { (2) } & L_{=}\left(z^{R}\right) \cap \partial R \quad \neq \emptyset
\end{array}
$$

The set of all optimal locations is in this case $L_{=}\left(z^{R}\right) \cap \partial R$

Since

$$
\begin{aligned}
L_{\leq}(z) & =\left\{Y: \max _{m=1, \ldots, M} l_{2}\left(E x_{m}, Y\right) \leq z\right\} \\
& =\left\{Y: l_{2}\left(E x_{m}, Y\right) \leq z \quad \forall m=1, \ldots, M\right\} \\
& =\bigcap_{m=1, \ldots, M}\left\{Y: l_{2}\left(E x_{m}, Y\right) \leq z\right\}
\end{aligned}
$$

we can write level sets as intersections of $M$ balls $B\left(E x_{m}, z\right)$ centered at the existing facilities $E x_{m}$, with radii $z$ :

$$
L_{\leq}(z)=\bigcap_{m=1, \ldots, M} B\left(E x_{m}, z\right)
$$

For the level curve we get:

$$
L_{=}(z)=\partial\left(\bigcap_{m=1, \ldots, M} B\left(E x_{m}, z\right)\right)
$$




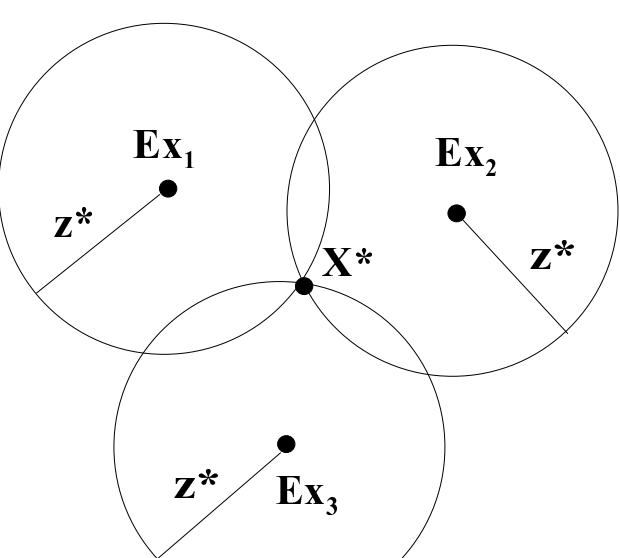

a)

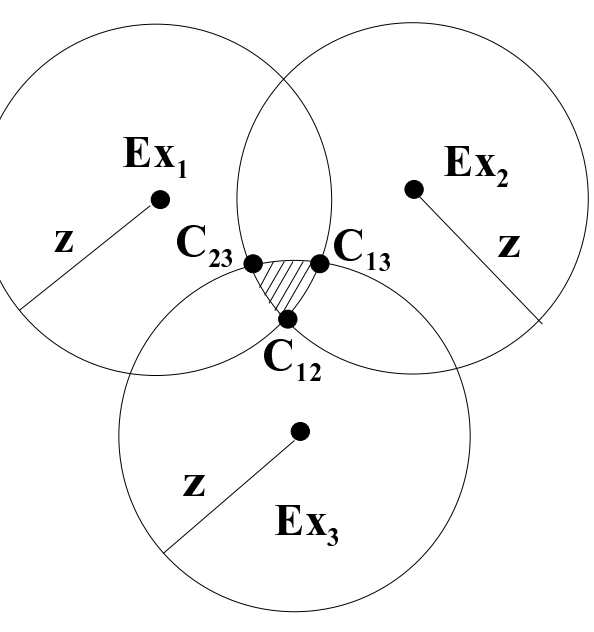

\section{Figure 1:}

a) The optimal location $X^{*}$ as unique intersection of $B\left(E x_{m}, z^{*}\right), m=1,2,3$

b) $L_{\leq}(z)$ is the shaded area with corner points $C_{12}, C_{13}$ and $C_{23}$.

The optimal value $z^{*}$ of the unrestricted problem is the smallest value $z$ with $L_{\leq}(z) \neq \emptyset$. In that case $L_{\leq}\left(z^{*}\right)=\left\{X^{*}\right\}$ (see Figure 1a). For $z>z^{*} L_{\leq}(z)$ is an area in the plane (see Figure 1b).

If $X^{*} \in \operatorname{int}(R)$ is not feasible for the restricted problem we need to increase $z^{*}$ until conditions (1) and (2) of Theorem 2.2 are satisfied. Due to the representation of $L_{\leq}(z)$ as intersection of the balls $B\left(E x_{m}, z\right)$, this may happen in two different situations shown in Figure 2 . 
a)
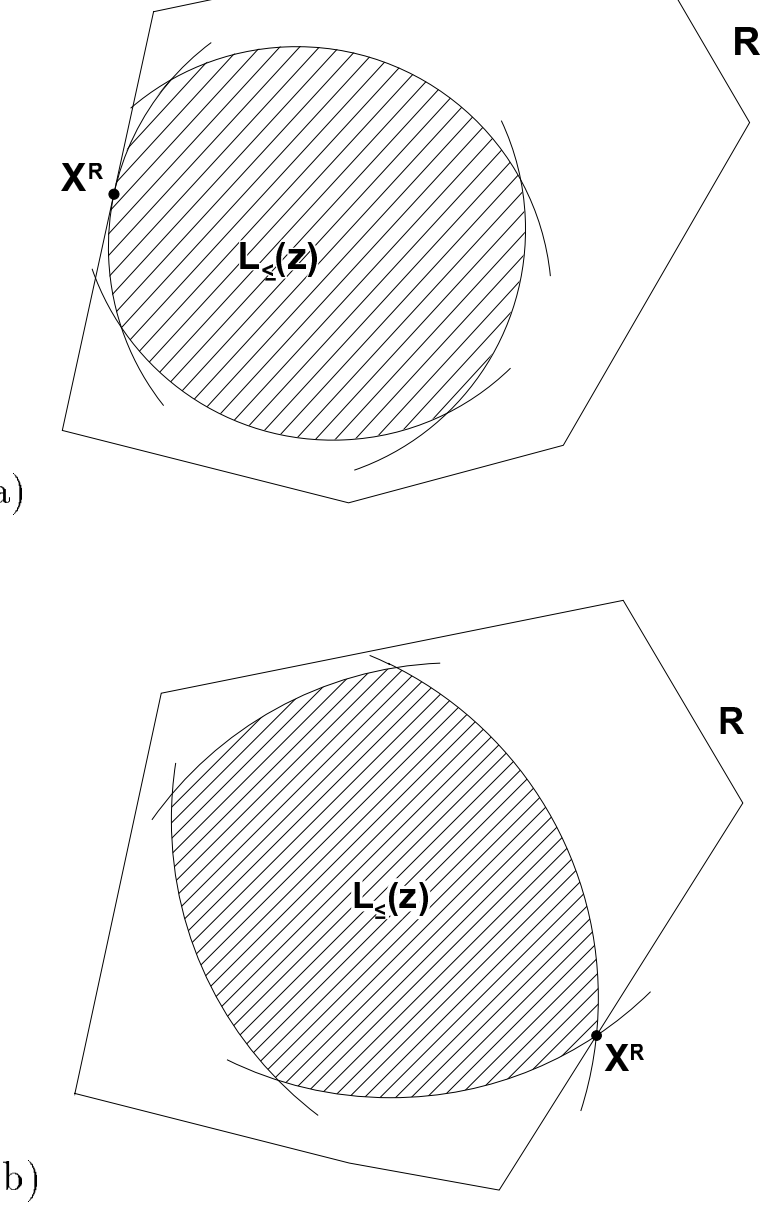

Figure 2: $\quad$ Two alternatives satisfiying conditions (1) and (2) of Theorem 2.2.

If the restricting set $R$ is a polyhedron with $Q$ facets $f_{1}, \ldots, f_{Q}$, we can therefore characterize optimal solutions for the restricted problem as follows.

Theorem 2.3 Let $X^{R}$ be an optimal solution of the restricted Euclidean center problem with objective value $z^{R}=\max _{m=1, \ldots, m} l_{2}\left(E x_{m}, X\right)$ and $z^{R}>z^{*}$. Then one of the following statements is correct:

a) $X^{R}$ is projection point $P_{m q}$ of one of the existing facilities $E x_{m}, m \in\{1, \ldots, M\}$, to one of the facets $f_{q}, q=1, \ldots, Q$ of $R$ (see Figure $2 a$ ).

b) $X^{R}$ is intersection point of two cycles $C\left(E x_{l}, z^{R}\right)$ and $C\left(E x_{k}, z^{R}\right)$ (a corner point $C_{l k}\left(z^{R}\right)$ ) lying on $\partial R$ (see Figure $\left.2 b\right)$ where $C\left(E x_{m}, z^{R}\right)=\partial\left(B\left(E x_{m}, z^{R}\right)\right) \forall m \in\{1, \ldots, M\}$ 
Proof. The result follows from Theorem 2.2 and the fact that $R$ is a polyhedron: $X^{R} \in \partial R$ and $O p t^{*} \cap \partial R=\emptyset$ implies that $\partial R$ and $L_{\leq}\left(z^{R}\right)$ are touching each other from within $R$. Therefore, one of the facets $f_{q}$ of $R$ is a tangent to one of the cycles $C\left(E x_{m}, z^{R}\right)$, and consequently $f_{q}$ and $C\left(E x_{m}, z^{R}\right)$ touch in $P_{m q}$, (Case(a)) or $X^{R}=C_{l k}\left(z^{R}\right)$ is corner point of $L_{\leq}\left(z^{R}\right)(\operatorname{Case}(\mathrm{b}))$.

\section{Polynomial Algorithm for Restricted Euclidean Cen- ter Problems}

Theorem 2.3 characterizes the candidates for being optimal locations of the restricted problem.

In Case (a) there are $M \cdot Q$ projection points of the existing facilities $E x_{m}, m=1, \ldots, M$ to the $Q$ facets of $R$.

For Case (b) we know from elementary geometry that the set of all corner points

$$
\left\{C_{l k}(z) \in C\left(E x_{l}, z\right) \cap C\left(E x_{k}, z\right): z \geq \frac{1}{2} l_{2}\left(E x_{l}, E x_{k}\right)\right\}
$$

can be represented by the perpendicular bisector $M_{l k}$ of $E x_{l}$ and $E x_{k}$. Therefore, possible candidates for $X^{R}$ are obtained by considering the intersection of $M_{l k}$ with $\partial R$. If the intersection contains two or less points, they are included in the candidate list. Otherwise, the perpendicular bisector $M_{l k}$ contains a complete facet $f_{q}$ of $R$. We have shown in the Appendix that, in this case, $M_{l k}$ can be dropped from further consideration.

For each candidate $X$ we compute its level $z=z(X)$ given by the radius of $C\left(E x_{m}, z\right)$ in Case (a) and the radii of $C\left(E x_{l}, z\right)$ and $C\left(E x_{k}, z\right)$ in Case (b). If $l_{2}\left(E x_{i}, X\right) \leq z$ for all $i=1, \ldots, M$, then $X$ is a feasible candidate, otherwise we drop $X$ from further consideration. The feasible candidate with the smallest $z(R)$ is an optimal location for $1 / P / v_{i}=1, R$ polyhedron $/ l_{2} / \max$. (Because $z^{R}>z^{*}$ it is only necessary to check candidates $X$ with $z(X)>z^{*}$.)

In summary we obtain the following algorithm.

\section{Algorithm for restricted Euclidean center problems}

Input: $\quad\left\{E x_{m}: m=1, \ldots, M\right\}$ set of existing facilities

$$
R \text { polyhedron with facets } f_{1}, \ldots f_{Q}
$$

Output: $O p t^{R}$ set of all optimal locations

1. Find the optimal location $X^{*}$ of the unrestricted problem.

2. If $X^{*} \notin \operatorname{int}(R)$ output $O p t^{R}=\left\{X^{*}\right\}$

Else: Define $O p t^{R}=\emptyset$ and goto 3 .

3. a) Cand $=\emptyset$ 
b) For all $m=1, \ldots, M$ do

For all $q=1, \ldots, Q$ do

Find projection point $P_{m q}$ of $E x_{m}$ onto $f_{q}$ (if it exists) and compute $z\left(P_{m q}\right)=l_{2}\left(P_{m q}, E x_{m}\right)$.

Set Cand := Cand $\cup\left\{\left(P_{m q}, z\left(P_{m q}\right)\right)\right\}$

c) For all $l>k$ do

If the perpendicular bisector $M_{l k}$ of $E x_{l}$ and $E x_{k}$ satisfies $1 \leq\left|\left(M_{l k} \cap \partial R\right)\right| \leq 2$ do Compute the (at most two) intersection points $I_{l k}^{i}, i=1,2$ of $M_{l k}$ with $\partial R$ and the corresponding radii

$$
z\left(I_{l k}^{i}\right):=l_{2}\left(E x_{l}, I_{l k}^{i}\right)=l_{2}\left(E x_{k}, I_{l k}^{i}\right), i=1,2
$$

and define Cand $:=$ Cand $\cup\left\{\left(I_{l k}^{i}, z\left(I_{l k}^{i}\right)\right), i=1,2\right\}$

4. Remove all $(P, z)$ from Cand for which $z \leq z^{*}$ or $l_{2}\left(E x_{m}, P\right)>z$ for some $m=1, \ldots, M$

5. Opt $t^{R}=\{P:(P, z) \in$ Cand \& $z$ is minimum $\}$

The complexity of the algorithm is dominated by step 1 (solving the unrestricted problem) and step 4 . The complexity of step 4 is $0\left(M^{3}\right)+0\left(M^{2} Q\right)$. If we solve the unrestricted problem with the Algorithm of Megiddo [Meg83], we get an overall complexity of $0\left(M^{3}\right)+$ $0\left(M^{2} Q\right)$.

\section{Extensions}

In this section we will discuss some extensions of the theory developed in Section 2 .

\subsection{Weights $v_{i} \neq 1$}

If general weights $v_{i}>0$ are allowed, the level sets are defined using the relation

$$
l_{2}\left(E x_{m}, Y\right) \leq \frac{z}{v_{m}}
$$

Consequently, we can write

$$
L_{\leq}(z)=\bigcap_{m=1, \ldots, M} B\left(E x_{m}, \frac{z}{v_{m}}\right)
$$

As before, the candidate set consists of all projection points and of all corner points (see Theorem 2.2. and Figure 2), even if the circles $B\left(E x_{m}, \frac{z}{v_{m}}\right)$ have different sizes. Therefore we have to check all projection points $P_{m q}$ from existing facilities $E x_{m}$ to any facet $f_{q}$ and all points $X$, which fulfill

$$
v_{m} l_{2}\left(E x_{m}, X\right)=v_{k} l_{2}\left(E x_{k}, X\right)
$$


for any pair $E x_{m}=\left(a_{m 1}, a_{m 2}\right)$ and $E x_{k}=\left(a_{k 1}, a_{k 2}\right)$ of existing facilites. Simple calculations show, that

$$
\left\{X \in \mathbb{R}^{2}: v_{m} l_{2}\left(E x_{m}, X\right)=v_{k} l_{2}\left(E x_{k}, X\right)\right\}=C\left(M P_{m k}, r_{m k}\right)
$$

is a circle around $M P_{m k}$ with radius $r_{m k}$, where

$$
\begin{aligned}
M P_{m k} & =\left(\frac{a_{m 1}-\mu^{2} a_{k 1}}{1-\mu^{2}}, \frac{a_{m 2}-\mu^{2} a_{k 2}}{1-\mu^{2}}\right) \\
r_{m k} & =\frac{\mu}{\left|1-\mu^{2}\right|} l_{2}\left(E x_{m}, E x_{k}\right) \text { and } \\
\mu & =\frac{v_{k}}{v_{m}}
\end{aligned}
$$

To get the corner points we have to calculate the intersection of $C\left(M P_{m k}, r_{m k}\right)$ with $\partial R$ for all $m<k, m, k \in \mathcal{M}$ and can proceed as shown in the algorithm. That means we change step 3c as follows:

\section{$\left.3 c^{\prime}\right)$ For all $l>k$ do}

Compute the (at most $2 Q$ ) intersection points $I_{l k}^{i}, i=1,2, \ldots, W$ of $C\left(M P_{l k}, r_{l k}\right)$ with $\partial R$ and the corresponding radii

$$
z\left(I_{l k}^{i}\right):=v_{l} l_{2}\left(E x_{l}, I_{l k}^{i}\right)=v_{k} l_{2}\left(E x_{k}, I_{l k}^{i}\right), i=1,2, \ldots, W
$$

and define Cand $:=$ Cand $\cup\left\{\left(I_{l k}^{i}, z\left(I_{l k}^{i}\right)\right), i=1,2, \ldots, W\right\}$

For abitrary weights $v_{i}$ that method does not work, because Theorem 2.1. does not hold. If all weights $v_{i} \leq 0$ we get the problem of obnoxious facility planning, that was treated for exampl e by [CHJT91], [DW80] or [CP95].

\subsection{Set of polyhedra as restricting set}

If $R=R_{1} \dot{\cup} \cdots \dot{\cup} R_{k}$ is the disjoint union of $K$ polyhedra the results of Sections 2 and 3 need only be slightly modified. If the optimal location $X^{*}$ of the unrestricted problem is not contained in any $\operatorname{int}\left(R_{k}\right), k=1, \ldots, K$, the restricted problem is trivially solved. Otherwise, there exists a unique $k$ such that $X^{*} \in \operatorname{int}\left(R_{k}\right)$ and the restricted problem is solved by replacing $R$ by $R_{k}$.

\subsection{Non-convex polyhedra as restricting set}

If $R$ is a polyhedron with extreme points $E x t=\left\{e_{1}, \ldots, e_{Q}\right\}$ we define:

$e_{q} \in E x t$ is an inner extreme point, if $e_{q} \in \operatorname{int}(\operatorname{conv}(E x t))$. For convex polyhedra the set of inner extreme points is empty.

Theorem 4.1 Let $X^{R}$ be an optimal solution of the restriced Euclidean center problem with a polyhedron as restricting set. Let $z^{R}$ be the corresponding objective value and $z^{R}>z^{*}$. Then one of the following statements is correct:

a) $X^{R}$ is a projection point $P_{m q}$ 
b) $X^{R}$ is a corner point $C_{l k}$

c) $X^{R}$ is an inner extreme point $e_{q}$

Proof. As in the proof of Theorem 2.3 we know that $\partial R$ and $L_{\leq}\left(z^{R}\right)$ are touching each other from within $R$. Because $R$ is a polyhedron, this can happen in projection points $P_{m q}$ or corner points $C_{l k}$ and, additionally in all inner extreme points $e_{q}$.

To solve $1 / P / v_{i}=1, R=$ non-convex polyhedron $/ l_{2} / \max$ we can use the algorithm of Section 3 with a small modification. We need to add step $3 \mathrm{~d}$ as follows:

3d) For all $q=1, \ldots, Q$ do

If $e_{q}$ is an inner extreme point calculate

$$
z\left(e_{q}\right)=\max _{m \in \mathcal{M}} l_{2}\left(E x_{m}, e_{q}\right)
$$

and define Cand $:=$ Cand $\cap\left\{\left(e_{q}, z\left(e_{q}\right)\right)\right\}$

\section{APPENDIX}

Lemma 4.2 Let $E x_{l}, E x_{k}$ be two existing facilities. If the perpendicular bisector $M_{l k}$ of $E x_{l}$ and $E x_{k}$ contains a facet $f_{q}$ of the restricted set $R$, then, for finding all possible candidates for the solution $X^{R}$ of the restricted problem, it is not necessary to consider $M_{l k}$.

Proof. Let $f_{q} \subseteq M_{l k}$ and consider a point $X \in f_{q} \subseteq M_{l k}$. Then we know that $l_{2}\left(E x_{l}, X\right)=l_{2}\left(E x_{k}, X\right)=: z$. If $X$ is feasible, then $l_{2}\left(E x_{m}, X\right) \leq z$ for all facilities $m \in \mathcal{M}$. We can suppose that for all $m \in \mathcal{M} \backslash\{l, k\} \quad l_{2}\left(E x_{m}, X\right)<z$, because if $l_{2}\left(E x_{m}, X\right)=z$ for any $m \in \mathcal{M} \backslash\{l, k\} X$ would be added to the candidate set when regarding $M_{l m}$. Because of that strict inequality the level set $L_{\leq}(z)$ is determined by the balls $B\left(E x_{l}, z\right)$ and $B\left(E x_{k}, z\right)$ in a neighbourhood $U=U(X)$ of $\bar{X}$, that means

$$
A:=L_{\leq}(z) \cap U=B\left(E x_{l}, z\right) \cap B\left(E x_{k}, z\right) \cap U
$$

Because $X$ is not optimal for the unrestricted problem and $M_{l k}$ separates $E x_{l}$ and $E x_{k}$ we get that $\operatorname{int}(A) \subseteq \operatorname{int}\left(L_{\leq}(z)\right)$ contains points of both sides of $M_{l k}$. Therefore we find a point $Y \in A \subseteq L_{\leq}(z), Y \notin R$, such that $L_{\leq}(z) \subseteq R$ does not hold and $X$ cannot be optimal for the restricted problem according to Theorem 2.2.

\section{References}

[CHJT91] Pey-Chun Chen, Pierre Hansen, Brigitte Jaumard, and Hoang Tuy. Weber's problem with attraction and repulsion. Technical Report 62-91, Rutgers Center for Operations Research, 1991. 
[CP95] E. Carrizosa and F. Plastria. Constrained single facility euclidean minimal covering problems. Technical Report BEIF/86, Vrije Universiteit Brüssel, 1995.

[DW80] B. Dasarathy and L.J. White. A maxmin location problem. Operations Research, 28(6):1985-1, 1980 .

[EH72] J. Elzinga and D.W. Hearn. Geometrical solutions for some minimax location problems. Transportation Science, 6(4):379-394, 1972.

[Ham95] H.W. Hamacher. Mathematische Lösungsverfahren für planare Standortprobleme. Vieweg Verlag, 1995.

[HN93] H.W. Hamacher and S. Nickel. Multicriteria planar location problems. Technical Report 243, Universität Kaiserslautern, 1993. accepted by EJOR.

[HN95] H.W. Hamacher and S. Nickel. Restricted planar location problems and applications. Naval Research Logistics, 42(6):967-992, 1995.

[HV95] D.W. Hearn and J. Vijay. Codes of geometrical algorithms for the (weighted) minimum circle problem. European Journal of Operations Research (ORSEP), 80(1):236-237, 1995 .

[Meg83] N. Megiddo. Linear-time algorithms for linear programming in $R^{3}$ and related problems. SIAM Journal of Computation, 12(4):759-776, 1983.

[Nic95] S. Nickel. Codes of geometrical algorithms for the (weighted) minimum circle problem. European Journal of Operations Research (ORSEP), 80(1):236-237, 1995 . 